\title{
Chemical solution synthesis and ferromagnetic resonance of epitaxial thin films of yttrium iron garnet
}

\author{
Irene Lucas,,${ }^{1,2}$ Pilar Jiménez-Cavero, ${ }^{1,2}$ J. M. Vila-Fungueiriño, ${ }^{3}$ Cesar Magén, $, 2,4,6$ Soraya Sangiao, ${ }^{2,4}$ José Maria de \\ Teresa, ${ }^{2,4,6}$ Luis Morellón, ${ }^{1,2}$ and Francisco Rivadulla ${ }^{7, *}$ \\ ${ }^{1}$ Instituto de Nanociencia de Aragón (INA), Universidad de Zaragoza, Mariano Esquillor, Edificio I+D, 50018 Zaragoza, Spain \\ ${ }^{2}$ Departamento Física de la Materia Condensada, Universidad de Zaragoza, Pedro Cerbuna 12, 50009 Zaragoza, Spain \\ ${ }^{3}$ Institut d'Électronique et des Systèmes (IES) UMR 5214, CNRS Université de Montpellier, 860 rue Saint Priest, 34095 Montpellier, France \\ ${ }^{4}$ Laboratorio de Microscopías Avanzadas (LMA) - Instituto de Nanociencia de Aragón (INA), Universidad de Zaragoza, \\ 50018 Zaragoza, Spain \\ ${ }^{5}$ Fundación ARAID, 50018 Zaragoza, Spain \\ ${ }^{6}$ Instituto de Ciencia de Materiales de Aragón (ICMA), Universidad de Zaragoza- CSIC, 50009 Zaragoza, Spain \\ ${ }^{7}$ Centro de Investigación en Química Biolóxica e Materiais Moleculares (CIQUS), and Departamento de Química-Física, \\ Universidade de Santiago de Compostela, 15782 Santiago de Compostela, Spain
}

(Received 13 September 2017; published 20 December 2017)

\begin{abstract}
We report the fabrication of epitaxial $\mathrm{Y}_{3} \mathrm{Fe}_{5} \mathrm{O}_{12}$ (YIG) thin films on $\mathrm{Gd}_{3} \mathrm{Ga}_{5} \mathrm{O}_{12}$ (111) using a chemical solution method. Cubic YIG is a ferrimagnetic material at room temperature, with excellent magneto-optical properties, high electrical resistivity, and a very narrow ferromagnetic resonance, which makes it particularly suitable for applications in filters and resonators at microwave frequencies. But these properties depend on the precise stoichiometry and distribution of $\mathrm{Fe}^{3+}$ ions among the octahedral/tetrahedral sites of a complex structure, which hampered the production of high-quality YIG thin films by affordable chemical methods. Here we report the chemical solution synthesis of YIG thin films, with excellent chemical, crystalline, and magnetic homogeneity. The films show a very narrow ferromagnetic resonance (long spin relaxation time), comparable to that obtained from highvacuum physical deposition methods. These results demonstrate that chemical methods can compete to develop nanometer-thick YIG films with the quality required for spintronic devices and other high-frequency applications.
\end{abstract}

DOI: 10.1103/PhysRevMaterials.1.074407

\section{INTRODUCTION}

Magneto-optical properties of magnetic ceramic garnets are responsible for their application in microwave and magnetooptical devices [1,2]. For example, they combine a net magnetic moment with good electric insulating properties, low dielectric losses, a narrow ferromagnetic resonance (FMR) linewidth related to small spin-wave damping, optical transparency in a wide frequency range, and a pronounced Faraday effect [3]. In cubic iron garnets of general formula $\mathrm{A}_{3} \mathrm{Fe}_{5} \mathrm{O}_{12}\left(I a \overline{3} d, \mathrm{O}_{h}{ }^{10}\right.$ space group 230$), \mathrm{Fe}^{3+}$ ions occupy the different octahedral:tetrahedral sites, in a precise 2:3 distribution [4]. In the case of $\mathrm{Y}_{3} \mathrm{Fe}_{5} \mathrm{O}_{12}$ (YIG), the $\mathrm{Fe}^{3+}$ sublattices order antiferromagnetically, with nonmagnetic $\mathrm{Y}^{3+}$ ions located at the dodecahedral sites. Therefore, it is the uncompensated $\mathrm{Fe}^{3+}$ at a tetrahedral site which is responsible for the ferrimagnetic state at room temperature, up to a Curie temperature $\sim 560 \mathrm{~K}[4,5]$. With the narrowest FMR linewidth and smallest damping parameter among magnetic garnets, YIG stands out as an exemplary synthetic material for microwave and magneto-optical applications [3,6-9]. A renewed interest in magnetic garnets has emerged with the development of spintronics, magnonics, and spin caloritronics in the last few years $[6,10-12]$. In these areas, the availability of epitaxial thin films with low magnetic damping is essential for most studies and applications.

In this regard, the standard method for YIG single-crystal fabrication, liquid phase epitaxy [3], and other bulk synthetic

*f.rivadulla@usc.es, irenelucas@unizar.es methods cannot be used due to the difficulties in controlling the layer thickness down to the nanometer range [13]. The development of physical techniques such as pulsed laser deposition (PLD) or sputtering allowed this problem to be overcome, producing high-quality epitaxial thin films of YIG of a few nanometers with low values of magnetization damping $[7,10,14,15]$. In this sense, chemical solution deposition offers important advantages regarding affordability and scalability for thin-film fabrication. Therefore, the development of simpler, cheaper, and more versatile techniques to chemically synthesize high-quality epitaxial YIG thin films has become a scientific and technological challenge of considerable relevance in the field.

In this paper, we report the chemical solution (water-based) deposition of high-quality epitaxial crystalline YIG thin films ( $\sim 15 \mathrm{~nm}$ thick) on (111) $\mathrm{Gd}_{3} \mathrm{Ga}_{5} \mathrm{O}_{12}$ (GGG), with very high structural, chemical, and magnetic homogeneity. These high-quality YIG thin films show magnetic properties comparable to those obtained using physical methods, and FMR, which demonstrates the good magnetic homogeneity of the films. The results presented here are an important step forward in the fabrication of YIG thin films by chemical methods with the quality required for spintronic studies and high-frequency applications.

\section{EXPERIMENTAL SECTION}

Thin films of YIG ( $a=12.376 \AA$ ) were deposited on top of GGG (111) substrates ( $a=12.383 \AA$ ) by polymer-assisted deposition (PAD) from a water-based precursor solution $[16,17]$. 
Individual solutions of $\mathrm{Y}$ and $\mathrm{Fe}$ were prepared by dissolving the corresponding nitrates in water with ethylenediaminetetraacetic acid (EDTA, 1:1 molar ratio) and polyethylenimine (PEI) (1:1 mass ratio to EDTA). Each individual solution was filtrated using Amicon filtration units (10 kDalton), and retained portions were analyzed by inductively coupled plasma (ICP). The solutions were then mixed according to the desired final stoichiometry and concentrated to a final value of $\sim 170 \mathrm{mM}$. This concentration was adjusted to obtain films in the 15-20-nm-thick range. $35 \mu \mathrm{L}$ of solution was spin coated (4000 rpm, $20 \mathrm{~s}$, with acceleration/deceleration ramps of $\left.250 \mathrm{rpm} / \mathrm{s}^{2}\right)$ on $(111) \mathrm{GGG}$ substrates $\left(5 \times 5 \mathrm{~mm}^{2}\right)$.

The samples were then annealed in air at different temperatures $\left(650^{\circ} \mathrm{C}-850^{\circ} \mathrm{C}\right)$ and cooled down at $3{ }^{\circ} \mathrm{C} / \mathrm{min}$ to avoid the formation of cracks. The films show excellent homogeneity over the whole substrate with only small imperfections at the corners. X-ray characterization was performed in a highresolution Bruker D8 Advance diffractometer. Diffraction patterns, reflectivity measurements, and reciprocal space maps were performed to ascertain the crystalline quality of the films. Local structure characterization was carried out by scanning transmission electron microscopy (STEM) in an FEI Titan 60300 operated at $300 \mathrm{kV}$ and equipped with a high-brightness Schottky field emission gun, a CETCOR probe aberration corrector from CEOS to provide a spatial resolution better than $1 \AA$ in STEM mode, and a Gatan Imaging Filter 866 ERS for spectroscopic analysis. $Z$ contrast imaging was carried out in high-angle annular dark field (HAADF) with a probe convergence angle of $25 \mathrm{mrad}$ and an inner collection angle of approximately $58 \mathrm{mrad}$. HAADF imaging was combined with electron energy loss spectroscopy (EELS) to analyze chemically the heterostructures by spectrum imaging (SI). In this case, both the inner angle for the HAADF detector and the spectrometer collection angle were $60 \mathrm{mrad}$. SI was performed with an energy dispersion of $0.5 \mathrm{eV}$ and a dwell time around $0.04 \mathrm{~s}$. The specimen analyzed was a lamella extracted from the sample by focused-ion-beam (FIB) milling in a FEI Helios Nanolab 600 using a $5-\mathrm{kV}$ ion beam for the final thinning to reduce the surface amorphization layer.

The magnetic properties of the films were determined in a superconducting quantum interference device (SQUID) magnetometer. For magnetization dynamics, ferromagnetic resonance experiments (FMR) were performed from 200 to $450 \mathrm{~K}$ in a Bruker EMX spectrometer operating at $9.4 \mathrm{GHz}(\mathrm{X}$ band), equipped with an ER 4102ST resonant cavity (TE102 mode). Broadband FMR experiments were performed at room temperature at frequencies between 2 and $8 \mathrm{GHz}$. An rf field is generated parallel to the long axis of the sample by a (a)

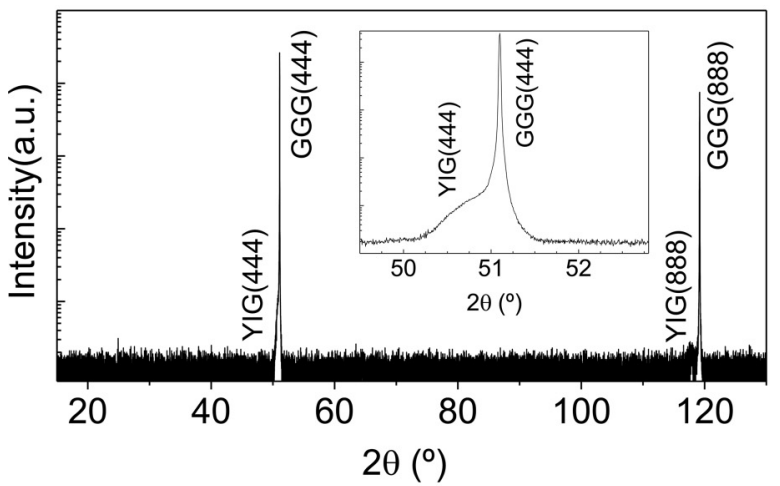

(b)

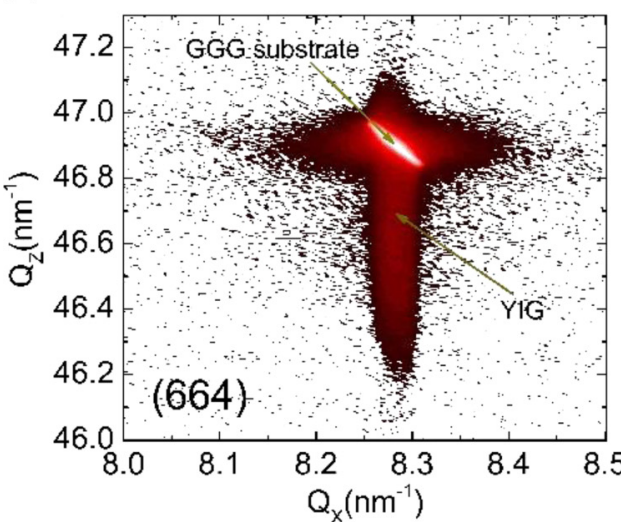

Counts (a.u.)

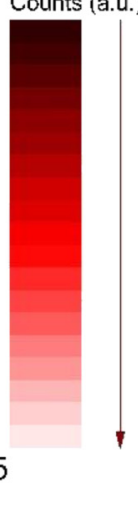

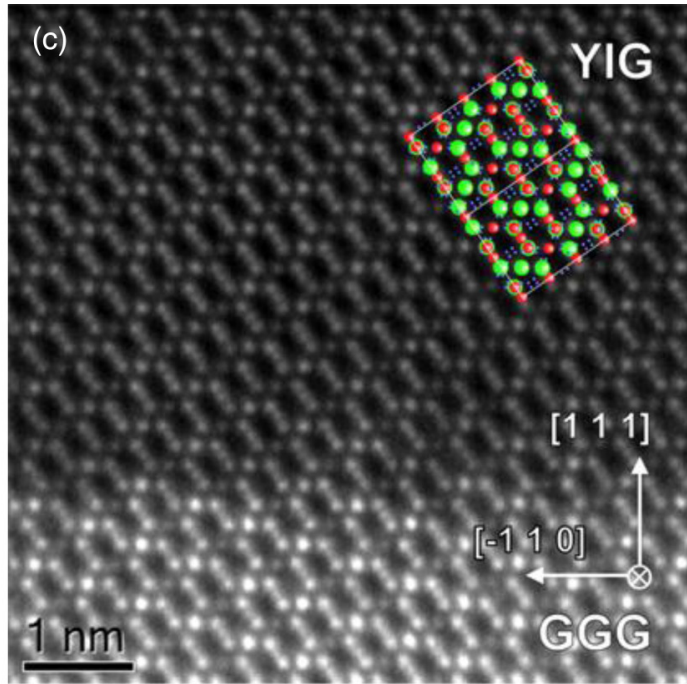

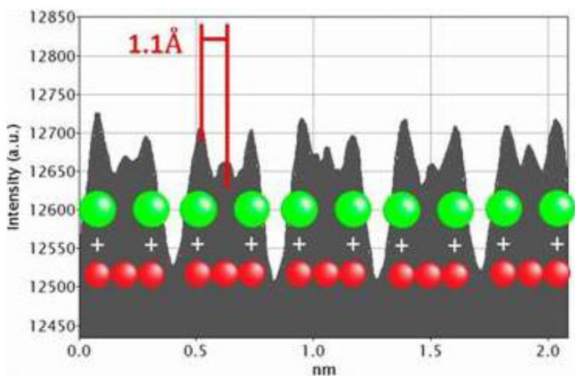

FIG. 1. (a) X-ray diffraction pattern of 15 -nm YIG on (111) GGG thin film synthesized at $750^{\circ} \mathrm{C}$. The x-ray pattern presents only the (444) and (888) Bragg peaks of YIG and GGG. No other phases are observed in the XRD scan from $2 \theta^{\circ}=15^{\circ}-130^{\circ}$. The inset shows a detailed scan around the (444) Bragg peak. (b) High-resolution reciprocal space map around the (664) Bragg reflection. (c) Cross-sectional HAADF-STEM image of the interface between the YIG film and the GGG (111) substrate, observed along the (002) zone axis. The sketch of the atomic structure of YIG is superimposed, where green, red, and blue balls represent Y, Fe, and O atoms, respectively. The EELS analysis taken along the arrow is shown in the panel under the picture. 
1-mm-wide shorted microstrip. The static magnetic field was applied in the film plane, perpendicular to the rf magnetic field.

\section{RESULTS AND DISCUSSION}

X-ray diffraction analysis shown in Figs. 1(a) and 1(b) confirms the oriented growth and good epitaxial matching to the substrate, as well as the absence of secondary phases, i.e., only the $(h h h)$ Bragg peaks from YIG and GGG are visible in the range $2 \theta^{\circ}=15^{\circ}-130^{\circ}$.

Epitaxial growth was further confirmed by HAADF imaging in STEM. A detail of the interface of the film with the GGG substrate is shown in Fig. 1(c), revealing a coherently strained film with an atomically sharp interface with the substrate. Geometrical phase analysis (GPA) of HAADF images in S1 (see Supplemental Material (SM) [18]) provides further proof of the fully strained growth, with a nearly perfect lattice match between film and substrate.

Chemical composition and homogeneity were studied by atomic resolution EELS, as shown in Fig. 1(c) (and Fig. $\mathrm{S} 1$ in the SM). No significant amount of vacancies or interatomic exchange was observed from the analysis. This complete structural and compositional analysis proves the good structural quality of the thin films obtained by PAD.

The dc magnetic field and temperature dependence of the magnetization were studied by SQUID magnetometry, and results are shown in Fig. 2. The best properties were obtained for the samples annealed in air at $750^{\circ} \mathrm{C}(2 \mathrm{~h})$, i.e., small coercive fields, $H_{C} \approx 2-3 \mathrm{Oe}$, large saturation magnetization $M_{S} \approx$ $140 \mathrm{emu} / \mathrm{cm}^{3}$ (around the theoretical value of $140 \mathrm{emu} / \mathrm{cm}^{3}$ at room temperature), and high Curie temperature, $T_{C} \sim 500 \mathrm{~K}$. These parameters are close to the values obtained in highquality crystals of YIG $[5,7,14]$ and suggest this to be the optimum temperature for the annealing of the films.

Thermal annealing under flowing oxygen at $750^{\circ} \mathrm{C}$ resulted in small segregation of iron oxides, small enough to become detectable by x-ray analysis, but worsening the magnetic properties of the films considerably. This is much better observed through the analysis of FMR of the films, as discussed below.

Previous reports of FMR of thin films of YIG deposited on (111) GGG by physical methods gave a large variety of results: Sun et al. [19] reported $\Delta H_{p p}$ between 5 and 17 Oe (X band) for films synthesized by PLD and damping constant $\alpha=2-3 \times 10^{-4}$. Hauser et al. [9] deposited the samples by PLD at room temperature and performed a postannealing under oxygen at high temperature for recrystallization. Following this strategy, they reported $\Delta H_{p p} \approx 1.5-4$ Oe and very small magnetization damping, with $\alpha=7 \times 10^{-5}$, similar to good single crystals [20]. On the other hand, Liu et al. [21] fabricated thin films by sputtering with $\Delta H_{p p} \approx 6-8$ Oe and $\alpha=0.9-1.3 \times 10^{-3}$. Onbasli et al. [7] obtained $\Delta H_{p p} \approx$ $3-4$ Oe and $\alpha=2 \times 10^{-4}$ for YIG deposited on (100) GGG by PLD. Our own samples fabricated by PLD in (111) GGG show $\Delta H_{p p} \approx 4-5$ Oe (see Fig. S2 in the SM).

The analysis of the FMR for the films of YIG synthesized by PAD in this work is shown in Fig. 3. For the samples prepared at $750^{\circ} \mathrm{C}$, a single sharp line with $\Delta H_{p p} \approx 17-20$ Oe is observed in the FMR spectrum [Fig. 3(a)]. Moving away from this temperature of synthesis results in a broadening of the resonance FMR line of YIG [Fig. 3(b)] and the appearance of extra peaks, which might be associated to the presence of small secondary phases (particularly those at higher resonance fields). These results were reproduced on various films and confirm that thermal annealing at $750{ }^{\circ} \mathrm{C}$ in air is the optimum condition for the preparation of YIG films by PAD.

The value of $\Delta H_{p p} \approx 17-20$ Oe for the PAD- $750^{\circ} \mathrm{C}$ films is like that of other films deposited by sputtering, and it is the best result reported to date for a YIG thin film grown by a chemical method. FMR analysis of different parts $(\approx 1 \times$ $1 \mathrm{~mm}^{2}$ ) of films deposited on $\approx 7 \times 7-\mathrm{mm}^{2}$-mm long substrates show similar linewidths in different parts of the crystal, demonstrating the good magnetic homogeneity of the films.

The in-plane magnetic anisotropy of the PAD-750 ${ }^{\circ} \mathrm{C}$ films was studied through the angular dependence of $H_{r}$ at $200 \mathrm{~K}$. The results are shown in the polar plot of Fig. 3(c). There is a threefold anisotropy of magnetocrystalline origin due to the symmetry induced in the film by the epitaxial growth on the (111) GGG. The well-defined angular dependence shows that the magnetic easy axis is highly oriented throughout the film. This is much better observed in the films deposited by PAD than PLD (see SM Fig. S3 and Ref. [19]). The reason is that contrary to PLD, films grown by PAD from a solution crystallize and grow from the substrate in conditions close to thermodynamic equilibrium, producing very clean interfaces and good epitaxial matching to the substrate.

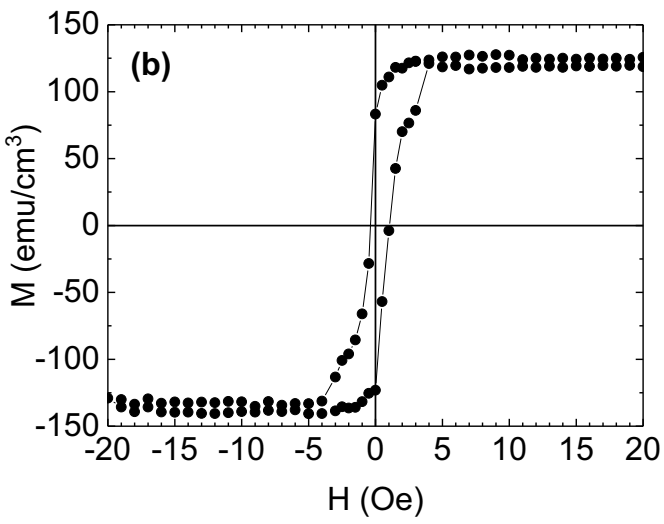

FIG. 2. (a) Temperature dependence of the magnetization of a 14-nm thin film of YIG synthesized at $750^{\circ} \mathrm{C}$ (open squares) after subtracting the contribution of the GGG substrate. The solid line shows the calculated Brillouin function for $J=5 / 2$ (high-spin Fe ${ }^{3+}$ ), $T_{C}=464 \mathrm{~K}$, and $M_{S}=140 \mathrm{emu} / \mathrm{cm}^{3}$. (b) $M(H)$ hysteresis curve of the same film taken at $290 \mathrm{~K}$, with $H_{C} \approx 1$ Oe and $M_{S} \approx 135 \mathrm{emu} / \mathrm{cm}^{3}$. 

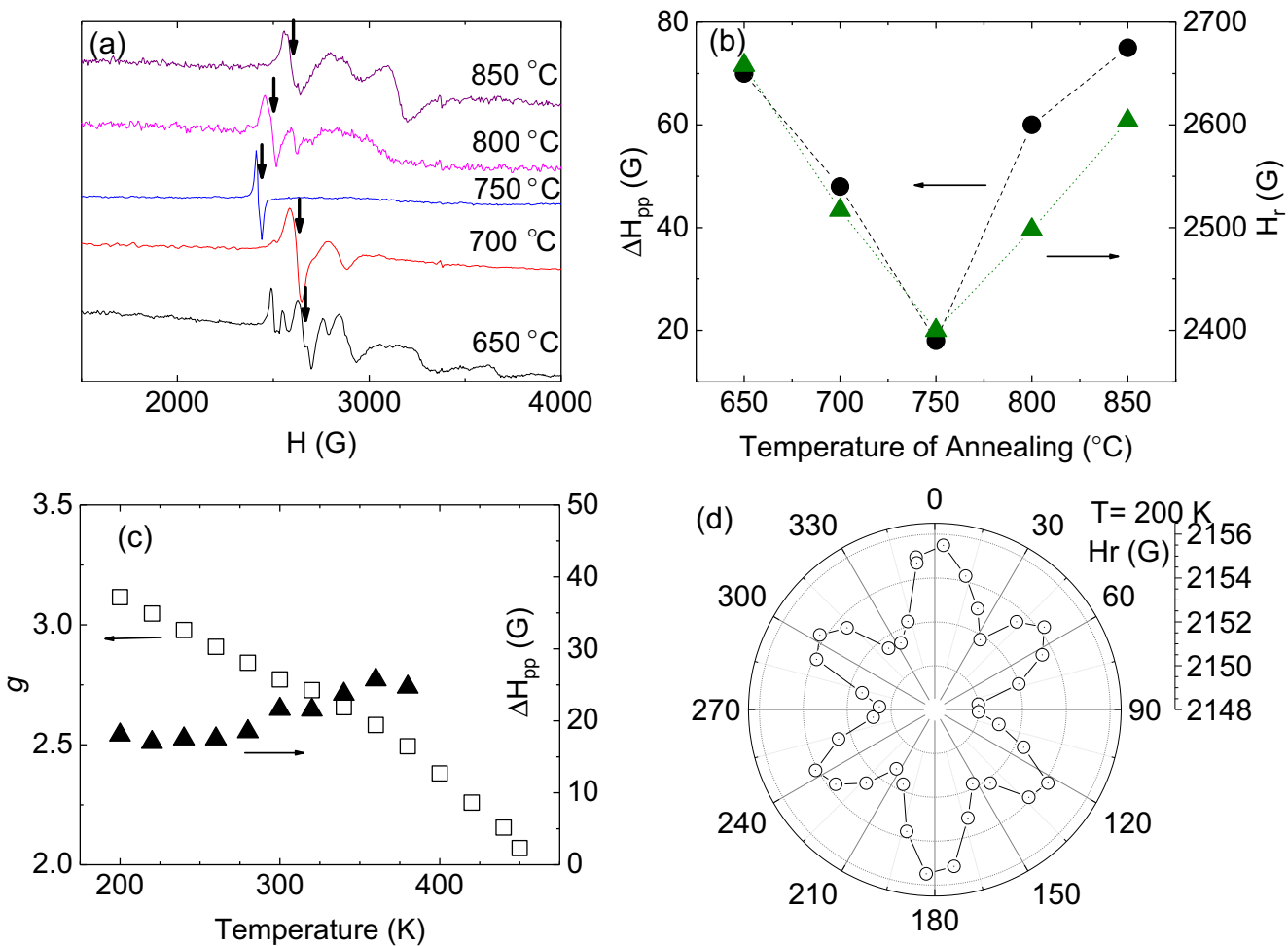

FIG. 3. (a) FMR spectra for different YIG samples depending on the annealing temperature. (b) Room-temperature peak-to-peak linewidth (solid triangles) and resonance field (solid circles) dependence on annealing temperature. (c) Temperature dependence of the $g$ factor obtained from $H_{r}$ and linewidth of the YIG-PAD- $750{ }^{\circ} \mathrm{C}$ film. The $g$ factor decreases towards the paramagnetic value of 2.0 at $\sim 450 \mathrm{~K}$, slightly lower than the $T_{C}$ determined from $M(H)$ measurements. (d) Angular dependence of the resonance field for the same film at $200 \mathrm{~K}$. The threefold anisotropy characteristic of the hexagonal symmetry of the (111) plane of YIG is clearly observed.

The water/PEI ratio and the heating ramp $\left(3^{\circ} \mathrm{C} / \mathrm{min}\right)$ were important parameters to minimize the presence of pores throughout the film and surface. Pores between grains and on the surface of the films produce demagnetizing fields which are an important source of line broadening. The extra linewidth broadening produced by porosity, $\Delta H^{*}{ }_{\mathrm{pp}}$, is proportional to $M$ (a demagnetization effect) and can be fitted to the following equation [22-24]:

$$
\Delta H_{p p}^{*}=(4 \pi M) p \phi
$$

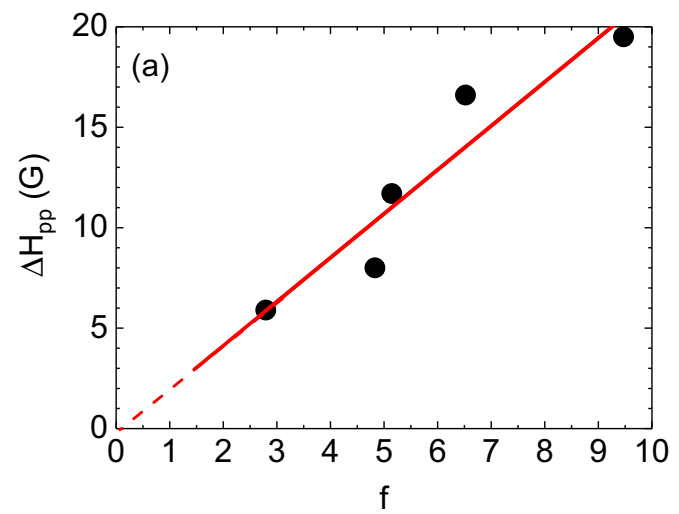

where $p$ is the porosity and $\phi$ is a factor related to the shape geometry of the pores. Under properly optimized conditions, the temperature dependence of $\Delta H_{p p}$ is very small [Fig. 3(c)], increasing slightly only as $T_{C}$ is approached, due to the increasing contribution of paramagnetic $\mathrm{Fe}^{3+}$ ions, indicating a low porosity. (See SM Fig. S4 for atomic force microscopy surface topography.)

On the other hand, when the annealing temperatures deviate from $750^{\circ} \mathrm{C}$ or when the amount of polymer is not properly adjusted, $\Delta H_{\mathrm{pp}}$ increases up to $40-60$ Oe and shows a

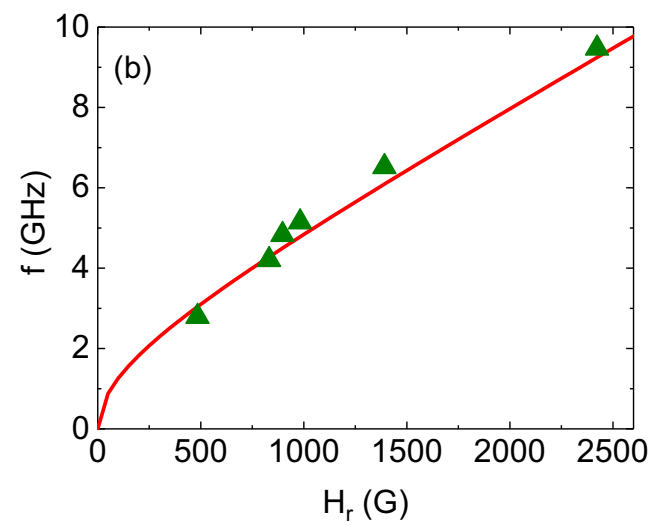

FIG. 4. (a) Peak-to-peak linewidth $\Delta H_{p p}$ vs frequency for a YIG film grown by PAD at $750^{\circ} \mathrm{C}$. The solid line is the linear fit. (b) Magnetic field dependence of the resonant frequency for the same sample. Solid curve is the fit to the data using the Kittel formula for $|\gamma| / 2 \pi=2.89 \mathrm{MHz} / \mathrm{Oe}$ and $M_{S}=144.2 \mathrm{emu} \mathrm{cm}^{-3}$. 
TABLE I. Comparison of magnetic data for thin films of YIG synthesized by PAD with other films deposited by physical methods.

\begin{tabular}{lcccc}
\hline \hline Deposition method & Thickness $(\mathrm{nm})$ & $M_{s}\left(\mathrm{emu} / \mathrm{cm}^{3}\right)$ & Gyromagnetic ratio $|\gamma| / 2 \pi(\mathrm{MHz} /$ Oe $)$ & Gilbert damping $(\alpha)$ \\
\hline PAD (this work) & 15 & 144 & 2.89 & $\approx 5 \times 10^{-3}$ \\
Sputtering [14] & 40 & 145 & & $\approx 7 \times 10^{-4}$ \\
Sputtering [20] & $10-26$ & 140 & 2.8 & $\approx 1 \times 10^{-3}$ \\
PLD [18] & $5-35$ & $133-149$ & 2.8 & $\approx 3.2 \times 10^{-4}$ \\
PLD [7] & $17-190$ & $120-137$ & & $\approx 2-7 \times 10^{-4}$ \\
LPE [13] & $83-113$ & 132 & $2.7-2.80$ & $\approx 2 \times 10^{-4}$ \\
PLD + recryst [9] & $20-56$ & 143 & & $\approx 6-8 \times 10^{-5}$ \\
\hline \hline
\end{tabular}

characteristic temperature dependence as $T_{C}$ is approached from low temperature (see Fig. S5 in the SM), indicating the contribution of porosity.

A larger surface porosity in the films deposited by PAD is the most probably reason for the slightly larger linewidth with respect to the samples synthesized by PLD (Fig. S2).

Broadband FMR experiments have been performed on optimized PAD- $750^{\circ} \mathrm{C}$ films. The dependence of the peakto-peak linewidth on the excitation frequency is presented in Fig. 4(a). The linear dependence of $\Delta H_{p p}$ is expected within the frame of the Landau-Lifshitz-Gilbert equation and allows for a straightforward determination of the intrinsic Gilbert damping coefficient $\alpha$ according to [25]

$$
\Delta H_{p p}=\Delta H_{p p}(0)+\frac{2 \alpha}{\sqrt{3}(|\gamma| / 2 \pi)} f .
$$

Here $\gamma$ denotes the gyromagnetic ratio, and $\Delta H_{p p}(0)$ is the extrinsic linewidth associated with the magnetic inhomogeneities within the material. To find the value of the intrinsic Gilbert damping parameter $\alpha$ from the linear fitting shown in Fig. 4(a) and Eq. (2), we determined the value of the gyromagnetic ratio in the samples. Figure 4(b) shows the magnetic field dependence of the resonant frequency fitted to the Kittel formula for the uniform precession mode [26]:

$$
f=\left(\frac{\gamma}{2 \pi}\right) \sqrt{H_{r}\left(H_{r}+4 \pi M_{s}\right)},
$$

where $M_{s}$ is the saturation magnetization of the YIG film. From the fit, the saturation magnetization is found to be $M_{s}=144.2 \mathrm{emu} \mathrm{cm}^{-3}$ and the gyromagnetic ratio $|\gamma| / 2 \pi=$ $2.89 \mathrm{MHz} / \mathrm{Oe}$. These values are comparable to the bulk YIG values (140 emu cm $\mathrm{cm}^{-3}$ and $2.80 \mathrm{MHz} / \mathrm{Oe}$ ). The Gilbert damping parameter calculated from these data $\alpha=5.50 \times 10^{-3}$. A comparison with some of the best results obtained from other methods taken from the literature is shown in Table I. Our values of $M_{s}$ and $\alpha$ compare very well with some of the best samples obtained by sputtering.

From the linear fitting shown in Fig. $4(\mathrm{a}), \Delta H_{p p}(0) \approx 0$, smaller than the experimental error. Therefore, any magnetic inhomogeneity does not seem to play a significant role in
FMR linewidth in YIG films fabricated by PAD under properly optimized conditions.

Finally, we have deposited YIG under optimized conditions of temperature and atmosphere, etc., on (001)- $\mathrm{Y}_{3} \mathrm{Al}_{5} \mathrm{O}_{12}$ (YAG) $(a=12.0 \AA)$. The X-ray diffraction (XRD) pattern shown in Fig. S6(a) (SM) shows the oriented growth of YIG films. However, the contamination of YAG substrates with paramagnetic impurities [probably $\mathrm{Fe}^{2+}$, see Figs. S6(b) and S6(c)] prevents an accurate analysis of the magnetic properties of the films.

\section{CONCLUSIONS}

In summary, we have demonstrated that epitaxial thin films of YIG can be fabricated by a chemical solution method with high crystalline quality and with ferromagnetic resonance properties suitable for spintronic and high-frequency applications. The magnetic properties are comparable to samples obtained by sputtering. This is an important advance for the fabrication of YIG films using an affordable and easily scalable method. Our results open new and promising possibilities about synthesizing high-quality nanometric-thick YIG films by using a low-cost, simple, and versatile chemical method.

\section{ACKNOWLEDGMENTS}

This work was supported by the Ministry of Science of Spain (Projects No. MAT2016-80762-R, MAT2017-82970C2-R, and No. MAT2014-51982-C2), the Consellería de Cultura, Educación e Ordenación Universitaria, Xunta de Galicia (ED431F 2016/008, and Centro Singular de Investigación de Galicia Accreditation 2016-2019, ED431G/09), the European Regional Development Fund (ERDF), and the Aragón Regional Government through Projects E26. The microscopy works have been conducted in the Laboratorio de Microscopías Avanzadas (LMA) at Instituto de Nanociencia de Aragón (INA), Universidad de Zaragoza. The authors acknowledge the LMA-INA for offering access to their instruments and expertise. Pilar Jiménez-Cavero acknowledges Spanish MECD for support through FPU program (Reference No. FPU014/02546).
[1] J. Douglas Adam, L. E. Davis, G. F. Dionne, E. F. Schloemann, and S. N. Stitzer, Ferrite devices and materials, IEEE Trans. Microw. Theory Techn. 50, 721 (2002).
[2] B. J. H. Stadler and T. Mizumoto, Integrated magneto-optical materials and isolators: A review, IEEE Photon. J. 6, 0600215 (2014). 
[3] E. J. J. Mallmann, A. S. B. Sombra, J. C. Goes, and P. B. A. Fechine, Yttrium iron garnet: Properties and applications review, Solid State Phenom. 202, 65 (2013).

[4] S. Geller and M. A. Gilleo, The crystal structure and ferrimagnetism of yttrium-iron garnet, $\mathrm{Y}_{3} \mathrm{Fe}_{2}\left(\mathrm{FeO}_{4}\right)_{3}$, J. Phys. Chem. Solids. 3, 30 (1957).

[5] E. E. Anderson, Molecular field and magnetization of YIG, Phys. Rev. 134, A1581 (1964).

[6] A. A. Serga, A. V. Chumak, and B. Hillebrands, YIG magnonics, J. Phys. D: Appl. Phys. 43, 264002 (2010).

[7] M. C. Onbasli, A. Kehlberger, D. H. Kim, G. Jakob, M. Kläui, A. V. Chumak, B. Hillebrands, and C. A. Ross, Pulsed laser deposition of epitaxial yttrium iron garnet films with low Gilbert damping and bulk-like magnetization, APL Mater. 2, 106102 (2014).

[8] H. Yu, O. d'Allivy Kelly, V. Cros, R. Bernard, P. Bortolotti, A. Anane, F. Brandl, R. Huber, I. Stasinopoulos, and D. Grundler, Magnetic thin-film insulator with ultra-low spin wave damping for coherent nanomagnonics, Sci. Rep. 4, 6848 (2014).

[9] C. Hauser, T. Richter, N. Homonnay, C. Eisenschmidt, M. Qaid, H. Deniz, D. Hesse, M. Sawicki, S. G. Ebbinghaus, and G. Schmidt, Yttrium iron garnet thin films with very low damping obtained by recrystallization of amorphous material, Sci. Rep. 6, 20827 (2016)

[10] N. S. Sokolov, V. V. Fedorov, A. M. Korovin, S. M. Suturin, D. A. Baranov, S. V. Gastev, B. B. Krichevtsov, K. Yu. Maksimova, A. I. Grunin, V. E. Bursian, L. V. Lutsev, and M. Tabuchi, Thin yttrium iron garnet films grown by pulsed laser deposition: Crystal structure, static, and dynamic magnetic properties, J. Appl. Phys. 119, 023903 (2016).

[11] J. Barker and G. E. W. Bauer, Thermal Spin Dynamics of Yttrium Iron Garnet, Phys. Rev. Lett. 117, 217201 (2016).

[12] H. Wang, C. Du, P. Chris Hammel, and F. Yang, Spin current and inverse spin Hall effect in ferromagnetic metals probed by $\mathrm{Y}_{3} \mathrm{Fe}_{5} \mathrm{O}_{12}$-based spin pumping. Appl. Phys. Lett. 104, 202405 (2014).

[13] C. Dubs, O. Surzhenko, R. Linke, A. Danilewsky, U. Brückner, and J. Dellith, Sub-micrometer yttrium iron garnet LPE films with low ferromagnetic resonance losses, J. Phys. D: Appl. Phys. 50, 204005 (2017).

[14] S. Li, W. Zhang, J. Ding, J. E. Pearson, V. Novosad, and A. Hoffmann, Epitaxial patterning of nanometer-thick $\mathrm{Y}_{3} \mathrm{Fe}_{5} \mathrm{O}_{12}$ films with low magnetic damping, Nanoscale 8, 388 (2016).
[15] H. Chang, P. Li, W. Zhang, T. Liu, A. Hoffmann, L. Deng, and M. Wu, Nanometer-thick yttrium iron garnet films with extremely low damping, IEEE Magn. Lett. 5, 6700104 (2014).

[16] Q. X. Jia, T. M. McCleskey, A. K. Burrell, Y. Lin, G. E. Collis, H. Wang, A. D. Q. Li, and S. R. Foltyn, Polymer assisted deposition of metal-oxide thin films, Nat. Mater. 3, 529 (2004).

[17] J. M. Vila-Fungueiriño, B. Rivas-Murias, B. RodriguezGonzalez, O. Txoperena, D. Ciudad, L. E. Hueso, M. Lazzari, and F. Rivadulla, Room-temperature ferromagnetism in thin films of $\mathrm{LaMnO}_{3}$ deposited by a chemical method over large areas, ACS Appl. Mater. Interfaces 7, 5410 (2015).

[18] See Supplemental Material at http://link.aps.org/supplemental/ 10.1103/PhysRevMaterials.1.074407 further analysis of TEM, AFM, and FMR data of the films, as well as the results of YIG films deposited on YAG.

[19] Y. Sun, Y.-Y. Song, H. Chang, M. Kabatek, M. Jantz, W. Schneider, M. Wu, H. Schultheiss, and A. Hoffmann, Growth and ferromagnetic resonance properties of nanometer-thick yttrium iron garnet films, Appl. Phys. Lett. 101, 152405 (2012).

[20] S. Klingler, H. Maier-Flaig, C. Dubs, O. Surzhenko, R. Gross, H. Huebl, S. T. B. Goennenwein, and M. Weiler, Gilbert damping of magnetostatic modes in a yttrium iron garnet sphere, Appl. Phys. Lett. 110, 092409 (2017).

[21] T. Liu, H. Chang, V. Vlaminck, Y. Sun, M. Kabatek, A. Hoffmann, L. Deng, and M. Wu, Ferromagnetic resonance of sputtered yttrium iron garnet nanometer films, J. Appl. Phys. 115, 17A501 (2014).

[22] F. Rivadulla, M. A. López-Quintela, L. E. Hueso, J. Rivas, M. T. Causa, C. Ramos, R. D. Sánchez, and M. Tovar, Electron-spinresonance line broadening around the magnetic phase transition in manganites, Phys. Rev. B. 60, 11922 (1999).

[23] E. Scholmann, Spin-wave analysis of ferromagnetic resonance in polycrystalline ferrites, J. Phys. Chem. Solids. 6, 242 (1958).

[24] M. Sparks, Ferromagnetic resonance porosity linewidth theory in polycrystalline insulators, J. Appl. Phys. 36, 1570 (1965).

[25] S. S. Kalarickal, P. Krivosik, J. Das, K. S. Kim, and C. E. Patton, Microwave damping in polycrystalline Fe-Ti-N films: Physical mechanisms and correlations with composition and structure, Phys. Rev. B 77, 054427 (2008).

[26] C. Kittel, Introduction to Solid State Physics (John Wiley \& Sons, New York, 1996). 